\title{
APRENDIZAGEM BASEADA EM PROBLEMAS COMO METODOLOGIA DE ENSINO NA FORMAÇÃO DOS ENFERMEIROS ${ }^{1}$
}

\author{
Wagner Maciel Sarmento, Universidade Federal de Campina Grande (UFCG), \\ waguinho_braga@hotmail.com \\ Gerlane Cristinne Bertino Véras, Universidade regional do Cariri (UFC), \\ gc.veras@ bol.com.br
}

\begin{abstract}
RESUMO
Objetivou-se avaliar os aspectos positivos da Aprendizagem Baseada em Problemas para a formação dos enfermeiros. Trata-se de uma revisão integrativa da literatura nas bases de dados LILACS E MEDLINE utilizando-se os descritores "educação em enfermagem AND aprendizagem baseada em problemas". A coleta de dados ocorreu em julho de 2017. Foram incluídas publicações disponíveis na íntegra, em língua portuguesa e publicados entre os anos de 2010 a 2017. Os critérios de exclusão foram teses e monografias, artigos em duplicata, e que não tratassem da temática proposta. Foram préselecionados 32 artigos, e após aplicação dos critérios pré-estabelecidos, foram selecionados seis artigos. Constatou-se vários aspectos positivos da utilização da Aprendizagem Baseada em Problemas como metodologia de ensino na formação dos enfermeiros, dentre eles destacam-se a autonomia do estudante, trabalho em equipe, habilidade de comunicação, desenvolvimento de um pensamento crítico, aumento da autoestima, entre outras. Pode-se constatar que a utilização da Aprendizagem Baseada em Problemas é de extrema importância para o desenvolvimento de competências no enfermeiro em formação, promovendo o seu protagonismo na (re)construção do seu conhecimento. Sugere-se que a Aprendizagem Baseada em Problemas seja adotada pelas instituições de ensino e docentes como prática pedagógica permanente na formação dos enfermeiros.
\end{abstract}

PALAVRAS-CHAVE: educação em enfermagem 1; enfermagem 2; aprendizagem baseada em problemas 3 .

\section{PROBLEM BASED LEARNING AS TEACHING METHODOLOGY ON NURSES FORMATION}

\begin{abstract}
The goal was to evaluate the positive aspects of Problem Based Learning for the training of nurses. It is an integrative review of the literature in the LILACS and MEDLINE databases using the descriptors "education in nursing AND problem based learning". Data collection happened in July 2017. Were included full publications, in portuguese and published between the years 2010 and 2017. The exclusion criterons were theses and monographs, duplicate articles, and that did not address the proposed theme. 32 articles were pre-selected, and after applying the pre-established criterions, five articles were selected. Several positive aspects of the use of Problem Based Learning as a teaching methodology in the training of nurses were

${ }^{1} \mathrm{O}$ presente trabalho não contou com apoio financeiro de nenhuma natureza para sua realização.
\end{abstract}


highlighted, such as student autonomy, teamwork, communication skills, critical thinking, increased self-esteem, among others. It can be verified that the use of Problem Based Learning is extremely important for the development of competencies in the nurse in training, promoting their role in the (re)building of his knowledge. It is suggested that Problem Based Learning be adopted by educational institutions and teachers as a permanent pedagogical practice in the training of nurses.

KEY WORDS: nursing education 1; Nursing 2; Problem-Based Learning 3.

\section{APRENDIZAJE BASADA EN PROBLEMAS COMO METODOLOGÍA DE ENSEÑANZA EN LA FORMACIÓN DE LOS ENFERMEROS}

\section{RESUMEN}

El objetivo es evaluar los aspectos positivos del Aprendizaje Basado en Problemas para la formación de los enfermeros. Se trata de una revisión integradora de la literatura de las bases de datos LILACS Y MEDLINE utilizando los identificadores "educación en enfermería AND aprendizaje basada en problemas". La recogida de datos se realizó en julio de 2017. Fueron incluidas las publicaciones disponibles en su totalidad, en lengua portuguesa y publicadas entre los años 2010 y 2017. Los criterios de exclusión fueron tesis y monografías y artículos duplicados que no tratasen de la temática propuesta. Fueran pre seleccionados 32 artículos, y después de la aplicación de los criterios pre establecidos, fueran seleccionados cinco artículos. Se constataron varios aspectos positivos de la utilización del Aprendizaje Basado en Problemas como metodología de enseñanza en la formación de los enfermeros, entre ellos destacan la autonomía del estudiante, trabajo en equipo, habilidad de comunicación, desarrollo de un pensamiento crítico y aumento de la autoestima, entre otras. Se puede constatar que la utilización del Aprendizaje Basado en Problemas es de extrema importancia para el desarrollo de competencias en el enfermero en formación, promoviendo su protagonismo en la (re) construcción de su conocimiento. Se sugiere que el Aprendizaje Basado en Problemas sea adoptado por las instituciones de enseñanza y docentes como práctica pedagógica permanente en la formación de los enfermeros.

PALABRAS CLAVES: educación en enfermería 1; enfermería 2; aprendizaje basado en problemas 3 .

\section{INTRODUÇÃO}

Os enfermeiros são responsáveis por desenvolver atividades de gerenciamento, assistenciais, de ensino, pesquisa e questões políticas, no entanto, contudo, para que isso aconteça de forma efetiva e eficaz estes profissionais necessitam de uma formação apropriada. 
$\mathrm{O}$ processo de ensino-aprendizagem na enfermagem ainda encontra-se muito fragmentado e centrado no modelo tecnicista e tradicional de ensino, no qual o professor é encarado como ator principal e os conteúdos são transmitidos de forma isolada (CHICHARO et al., 2016). No entanto, Para atender as reais necessidades de saúde da população, as instituições de ensino precisam adaptar suas práticas pedagógicas a um modelo disciplinar que se afaste do puramente biológico e se adeque às mudanças paradigmáticas, permitindo formar profissionais habilitados para trabalhar em equipe, com vista a integralidade, interdisciplinaridade, humanização, qualidade e competência (BALLARIN et al., 2013).

Frente a necessidade de uma nova prática pedagógica, aponta-se a metodologia ativa de ensino. Essa metodologia possibilita a participação ativa do discente na construção do conhecimento, tornando-o protagonista do seu processo educativo (SEBOLD et al., 2010). Geralmente baseia-se em problemas e entre ela destaca-se a Metodologia da Problematização (MP) e Aprendizagem Baseada em Problemas (ABP) (SOBRAL; CAMPOS, 2012).

A ABP é uma metodologia que envolve a problematização de situações da realidade para serem explorados pelos discentes, estes, por sua vez, assumem a responsabilidade sobre o seu processo de aprendizagem, mediado pelo tutor, e, desse modo, são instigados a construir e reconstruir seus conhecimentos de forma autônoma, colaborativa, e critica-reflexiva (CAMPOS; RIBEIRO; DEPES, 2014)

Ante ao exposto, tendo em vista a necessidade de se repensar as políticas pedagógicas na enfermagem, essa pesquisa faz-se relevante e objetiva avaliar os aspectos positivos da Aprendizagem Baseada em Problemas para a formação dos enfermeiros.

\section{MATERIAIS E MÉTODOS}

Trata-se de uma revisão integrativa da literatura que envolveu as seguintes etapas: estabelecimento de hipóteses ou questões de pesquisa; amostragem ou busca na literatura; categorização dos estudos; avaliação dos estudos incluídos na revisão; interpretação dos resultados e síntese do conhecimento ou apresentação dos resultados.

Foi realizada nas bases de dados Literatura Latino-Americana e do Caribe em Ciências da Saúde (LILACS) e na Literatura Internacional em Ciências (MEDLINE), utilizando-se os descritores "educação em saúde" e "aprendizagem baseada em problemas" cadastrados nos 
Descritores de Ciências da Saúde (DeCS), associados através do operador booleano “AND”, no intuito de responder a questão norteadora "Quais as vantagens de utilizar a Aprendizagem Baseada em Problemas na formação do enfermeiro?". A coleta de dados ocorreu em julho de 2017. Foram incluídos artigos científicos disponíveis na íntegra, na língua portuguesa e publicados entre os anos de 2010 a 2017. Os critérios de exclusão foram outros documentos, como teses e monografias, artigos em duplicata, e que não tratassem da temática proposta. A pré-seleção dos textos se deu após leitura dos títulos e resumos dos artigos, que foram, então, selecionados após leitura detalhada de todo os textos, analisando se respondiam a questão norteadora do estudo.

A avaliação das informações relevantes de cada artigo foram sintetizadas utilizando-se um instrumento validado por Ursi (2005) para a extração dos dados, sendo catalogados em ordem crescente em relação ao ano de publicação dos artigos.

\section{RESULTADOS E DISCUSSÃO}

Foram pré-selecionados 32 estudos, e após aplicação dos critérios de inclusão e exclusão, e da leitura detalhada, selecionou-se seis artigos para compor a amostra do estudo, sendo cinco provenientes da LILACS e um da MEDLINE.

Segundo Marin et al. (2010), tanto a problematização como a ABP, são metodologias ativas de aprendizagem que auxiliam a superar o modelo tradicional de ensino pois possibilita que as disciplinas sejam trabalhadas de forma simultânea e harmônica, estimula o trabalho em equipe e permite a integração entre os ciclos básico e clínico e entre as dimensões biopsicossociais, favorecendo a formação de profissionais de saúde para o SUS, dos quais os enfermeiros fazem parte.

De acordo com Lira e Lopes (2011) a ABP, como metodologia de ensino sobre diagnósticos de enfermagem contribui para a construção de um pensamento crítico e a formação de enfermeiros capazes de raciocinar clinicamente, com poder de julgamento diagnóstico e, consequentemente, preparados para tomar decisões pertinentes.

Essa metodologia, Segundo Campos; Ribeiro e Depes (2014), objetiva interligar a teoria com a prática, pois ao interpor exemplos fictícios de problemas reais aos 
conhecimentos prévios dos discentes de enfermagem permite aproximá-los da realidade, contribuindo, dessa forma, para uma aprendizagem efetiva.

Giribet e Moya (2014) também encontraram na ABP vários pontos positivos que favorecem a formação dos enfermeiros, isto porque, acredita-se que quando os discentes são formados em um contexto que lhes permite autonomia na aprendizagem, como faz a ABP, terão repercussão positiva em sua futura profissão, já que essa metodologia pode favorecer o desenvolvimento de competências essenciais à sua prática profissional, por exemplo a formação de um pensamento crítico, habilidade de comunicação, trabalho em equipe, sentimento de segurança, aumento da autoestima em relação a profissão escolhida e motivação na aprendizagem percebida pelo desejo de buscar, ampliar e atualizar seus conhecimentos.

Dentre as competências proporcionadas pela ABP, Cogo et al. (2010) destaca sua contribuição para o desenvolvimento e organização do trabalho em grupo e, consequentemente, a construção de um conhecimento coletivo, o qual favorece a formação de profissionais familiarizados com as relações interpessoais e cooperação nas atividades, necessárias ao seu futuro processo de trabalho.

Campos; Ribeiro e Depes (2014) também inferiram que a ABP, oportuniza a autonomia do indivíduo diante do seu processo de formação, pois ao desenvolverem as atividades que essa metodologia propõe, os discentes passam a ter responsabilidade direta sobre o seu próprio aprendizado, ou seja, aprendem de forma independente e contínua e buscam aprimorar, construir e reconstruir seus conhecimentos assim como aplicá-los a realidade.

Mais um aspecto positivo da ABP é a possibilidade de associá-la a outras metodologias de ensino com o objetivo de reforçar o processo pedagógico. Machado; Göttems e Pires (2013) apontam que a ABP associada a arte, como a produção videográfica, possibilita ampliar e facilitar a aprendizagem na formação dos enfermeiros e demais profissionais de saúde com um pensamento crítico e reflexivo. Cogo et al. (2010), também perceberam que a associação dessa metodologia com tecnologias digitais favorece esse processo de ensino-aprendizagem, o qual coloca o aluno como protagonista na construção do conhecimento e o professor como facilitador desse processo. 
Quadro 1 - Especificações dos estudos analisados quanto ao título, autor, tipo de estudo e resultados.

\begin{tabular}{|c|c|c|c|c|}
\hline Autor/Ano & Título do artigo & Objetivo & Tipo de estudo & Resultados \\
\hline MARIN, 2010 & $\begin{array}{c}\text { Aspectos das } \\
\text { fortalezas e } \\
\text { fragilidades no uso } \\
\text { das Metodologias } \\
\text { Ativas de } \\
\text { Aprendizagem. }\end{array}$ & $\begin{array}{l}\text { Analisar as } \\
\text { fortalezas e } \\
\text { fragilidades } \\
\text { dos métodos } \\
\text { ativos de } \\
\text { aprendizagem na } \\
\text { ótica dos } \\
\text { estudantes. }\end{array}$ & $\begin{array}{l}\text { Estudo } \\
\text { qualitativo. }\end{array}$ & $\begin{array}{c}\text { Foi percebido pelos } \\
\text { estudantes que a } \\
\text { metodologia ativa, } \\
\text { especialmente a } \\
\text { problematização e a } \\
\text { ABP promovia a } \\
\text { integração dos ciclos } \\
\text { básicos e clínicos, } \\
\text { aspectos } \\
\text { biopsicossociais entre } \\
\text { outros como pontos } \\
\text { positivos e como } \\
\text { aspectos negativos } \\
\text { destacou-se a } \\
\text { dificuldade na busca de } \\
\text { conhecimentos } \\
\text { teóricos. }\end{array}$ \\
\hline $\begin{array}{c}\text { COGO et al., } \\
2010\end{array}$ & $\begin{array}{c}\text { Aprendizagem de } \\
\text { sinais vitais } \\
\text { utilizando } \\
\text { objetos } \\
\text { educacionais } \\
\text { digitais: opinião de } \\
\text { estudantes de } \\
\text { enfermagem }\end{array}$ & $\begin{array}{c}\text { Conhecer as } \\
\text { opiniões de } \\
\text { estudantes de } \\
\text { enfermagem } \\
\text { quanto à prática } \\
\text { pedagógica } \\
\text { sobre } \\
\text { sinais vitais } \\
\text { fundamentada } \\
\text { na aprendizagem } \\
\text { baseada em } \\
\text { problemas }\end{array}$ & $\begin{array}{l}\text { Estudo de } \\
\text { caso }\end{array}$ & $\begin{array}{c}\text { A atividade } \\
\text { desenvolvida no } \\
\text { ambiente virtual } \\
\text { associada a ABP foi } \\
\text { avaliada positivamente } \\
\text { pelos estudantes, sendo } \\
\text { considerada uma } \\
\text { metodologia nova, } \\
\text { interessante e } \\
\text { proveitosa. }\end{array}$ \\
\hline $\begin{array}{c}\text { LIRA; } \\
\text { LOPES, } 2011\end{array}$ & $\begin{array}{l}\text { Diagnóstico de } \\
\text { enfermagem: } \\
\text { estratégia educativa } \\
\text { fundamentada na } \\
\text { aprendizagem } \\
\text { baseada em } \\
\text { problemas }\end{array}$ & $\begin{array}{c}\text { Avaliar a } \\
\text { eficácia de uma } \\
\text { estratégia de } \\
\text { ensino sobre } \\
\text { diagnósticos } \\
\text { de enfermagem, } \\
\text { fundamentada } \\
\text { na } \\
\text { aprendizagem, } \\
\text { baseada em } \\
\text { problemas no } \\
\text { desempenho do } \\
\text { raciocínio } \\
\text { clínico e } \\
\text { julgamento } \\
\text { diagnóstico dos } \\
\text { discentes de } \\
\text { graduação }\end{array}$ & $\begin{array}{c}\text { Estudo } \\
\text { experimental }\end{array}$ & $\begin{array}{c}\text { O grupo que recebeu } \\
\text { intervenções da ABP } \\
\text { se destacou em relação } \\
\text { ao grupo que recebeu } \\
\text { intervenções de ensino } \\
\text { padrão. }\end{array}$ \\
\hline
\end{tabular}




\begin{tabular}{|c|c|c|c|c|}
\hline $\begin{array}{l}\text { MACHADO; } \\
\text { GÖTTEMS; } \\
\text { PIRES, } \\
2013\end{array}$ & $\begin{array}{l}\text { Aprendizagem em } \\
\text { saúde mental por } \\
\text { meio da produção } \\
\text { videográfica: relato } \\
\text { de experiência }\end{array}$ & $\begin{array}{c}\text { Descrever a } \\
\text { proposta } \\
\text { metodológica do } \\
\text { módulo de saúde } \\
\text { do adulto, com } \\
\text { ênfase na } \\
\text { avaliação de } \\
\text { aprendizagem }\end{array}$ & $\begin{array}{c}\text { Relato de } \\
\text { experiência }\end{array}$ & $\begin{array}{c}\text { A produção } \\
\text { videográfica foi } \\
\text { considerada pelos } \\
\text { estudantes uma } \\
\text { experiência válida para } \\
\text { a construção do } \\
\text { conhecimento coletivo } \\
\text { e sua associação com a } \\
\text { ABP estimula a } \\
\text { criatividade. }\end{array}$ \\
\hline $\begin{array}{l}\text { CAMPOS; } \\
\text { RIBEIRO; } \\
\text { DEPES, } \\
2014\end{array}$ & $\begin{array}{l}\text { Autonomia do } \\
\text { graduando em } \\
\text { enfermagem na } \\
\text { (re)construção do } \\
\text { conhecimento } \\
\text { mediado pela } \\
\text { aprendizagem } \\
\text { baseada em } \\
\text { problemas }\end{array}$ & $\begin{array}{c}\text { Analisar como a } \\
\text { Aprendizagem } \\
\text { Baseada em } \\
\text { Problemas } \\
\text { (ABP) promove } \\
\text { o } \\
\text { desenvolvimento } \\
\text { da autonomia do } \\
\text { aluno no } \\
\text { processo de } \\
\text { aprender a } \\
\text { aprender }\end{array}$ & $\begin{array}{c}\text { Estudo } \\
\text { exploratório }\end{array}$ & $\begin{array}{c}\text { Com a ABP os } \\
\text { estudantes aprenderam } \\
\text { de forma autonomia a } \\
\text { (re)construir e seus } \\
\text { conhecimentos. }\end{array}$ \\
\hline $\begin{array}{c}\text { GIRIBET; } \\
\text { MOYA, } 2014\end{array}$ & $\begin{array}{l}\text { Pontos fortes e } \\
\text { deficiências da } \\
\text { Aprendizagem } \\
\text { Baseada em } \\
\text { Problemas } \\
\text { sob a perspectiva } \\
\text { profissional de } \\
\text { enfermeiras }\end{array}$ & $\begin{array}{l}\text { Identificar os } \\
\text { pontos fortes e } \\
\text { as deficiências } \\
\text { na competência } \\
\text { percebidos pelas } \\
\text { enfermeiras } \\
\text { formadas com } \\
\text { um currículo } \\
\text { totalmente } \\
\text { constituído por } \\
\text { competências } \\
\text { resultantes da } \\
\text { Aprendizagem } \\
\text { Baseada em } \\
\text { Problemas }\end{array}$ & $\begin{array}{l}\text { Estudo de } \\
\text { Caso. }\end{array}$ & $\begin{array}{c}\text { Percebeu-se que as } \\
\text { enfermeiras formadas a } \\
\text { partir da ABP, } \\
\text { possuíam uma maior } \\
\text { capacidade de analisar } \\
\text { e resolver problemas, } \\
\text { além de possibilitar } \\
\text { outras competências } \\
\text { como pontos fortes e } \\
\text { como fragilidade } \\
\text { destacou-se a falta de } \\
\text { conhecimentos } \\
\text { teóricos. }\end{array}$ \\
\hline
\end{tabular}

Fonte: Elaborado pelos autores

\section{CONSIDERAÇÕES FINAIS}

A Aprendizagem Baseada em Problemas, assim como outros tipos de metodologias ativas, possuem vários aspectos positivos, portanto sua contribuição é salutar para a formação de profissionais enfermeiros, pois faz com que o discente apresente-se como protagonista na (re)construção do seu conhecimento, contribuindo para o desenvolvimento de suas competências com pensamento crítico, capacidade de análise, julgamento e soluções. 
Com isto, sugere-se que a Aprendizagem Baseada em Problemas seja adotada pelos docentes e instituições de ensino, como prática pedagógica na formação dos enfermeiros, visto a ampla gama de benefícios que oportuniza ao estudante e futuro profissional. Podendo ainda ser associada a outras metodologias como forma de reforçar o processo de ensino aprendizagem.

\section{REFERÊNCIAS BIBLIOGRÁFICAS}

CHICARO, S.C.R. et al., Fatores facilitadores do ensino-aprendizagem na formação do enfermeiro: uma revisão integrativa. fundam. care. Online, v. 8, n. 2, p. 4099-4108, abr-jun 2016.

SEBOLD, L. F. et al., metodologias ativas: uma inovação na disciplina de fundamentos para o cuidado profissional de enfermagem. Cogitare Enferm, v. 15, n. 4, p. 753-756, out-dez 2010. SOBRAL, F. R.; CAMPOS, C. J. G. Utilização de metodologia ativa no ensino e assistência de enfermagem na produçãonacional: revisão integrativa. Rev Esc Enferm USP v. 46, n. 1, p. 208-218, 2012.

BALLARIN, M. L. G. S. et al., Metodologia da problematização no contexto das disciplinas práticas terapêuticas supervisionadas. Cad. Ter. Ocup. UFSCar, São Carlos, v. 21, n. 3, p. 609-616, 2013.

CAMPOS, L. R. G. I.; RIBEIRO, M. R. R.; DEPES, V. B. D. Autonomia do graduando em enfermagem na (re)construção do conhecimento mediado pela aprendizagem baseada em problemas. Rev Bras Enferm, v. 67, n. 5, p. 818-824, set-out 2014.

LIRA, A. L. B. C.; LOPES, M.V.O. Diagnóstico de enfermagem: estratégia educativa fundamentada na aprendizagem baseada em problemas. Rev. Latino-Am. Enfermagem, v. 19, n. 4, p. 1-8, jul-ago 2011.

GIRIBET, M. C.; MOYA, J. L. M. Pontos fortes e deficiências da Aprendizagem Baseada em Problemas sob a perspectiva profissional de enfermeiras. Rev. Latino-Am. Enfermagem, v. 22, n. 5, p. 724-730 set.-out 2014. 
COGO, A. L. P. et al., aprendizagem de sinais vitais utilizando objetos educacionais digitais: opinião de estudantes de enfermagem. Rev Gaúcha Enferm, Porto Alegre, v. 31, n. 3, p. 435441 , set 2010.

MACHADO, D. M.; GÖTTEMS, L. B. D.; PIRES, M. R. G. M. aprendizagem em saúde mental por meio da produção videográfica: relato de experiência. Texto Contexto Enferm, Florianópolis, v. 22, n. 4, p. 1205-1213, Out-Dez 2013.

URSI, E. S. Prevenção de lesões de pele no perioperatório: revisão integrativa da literatura. [dissertação]. Ribeirão Preto: Universidade de São Paulo, Escola de Enfermagem de Ribeirão Preto; 2005.

MARIN, M. J. S. Aspectos das fortalezas e fragilidades no uso das Metodologias Ativas de Aprendizagem. Revista brasileira de educação médica. V. 34 n. 1 p. 13-20, 2010. 